\title{
Desmoglein-1 regulates esophageal epithelial barrier function and immune responses in eosinophilic esophagitis
}

\author{
JD Sherrill $^{1}, \mathrm{~K} \mathrm{KC}^{1}$, D Wu ${ }^{1}, \mathrm{Z}$ Djukic $^{2}$, JM Caldwell ${ }^{1}$, EM Stucke ${ }^{1}$, KA Kemme ${ }^{1}$, MS Costello ${ }^{1}$,

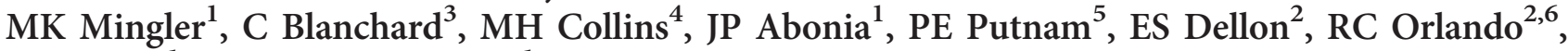 \\ SP Hogan ${ }^{1}$ and ME Rothenberg ${ }^{1}$
}

The desmosomal cadherin desmoglein-1 (DSG1) is an essential intercellular adhesion molecule that is altered in various human cutaneous disorders; however, its regulation and function in allergic disease remains unexplored. Herein, we demonstrate a specific reduction in DSG1 in esophageal biopsies from patients with eosinophilic esophagitis (EoE), an emerging allergic disorder characterized by chronic inflammation within the esophageal mucosa. Further, we show that DSG1 gene silencing weakens esophageal epithelial integrity, and induces cell separation and impaired barrier function (IBF) despite high levels of desmoglein-3. Moreover, DSG1 deficiency induces transcriptional changes that partially overlap with the transcriptome of inflamed esophageal mucosa; notably, periostin (POSTN), a multipotent proinflammatory extracellular matrix molecule, is the top induced overlapping gene. We further demonstrate that IBF is a pathological feature in EoE, which can be partially induced through the downregulation of DSG1 by interleukin-13 (IL-13). Taken together, these data identify a functional role for DSG1 and its dysregulation by IL-13 in the pathophysiology of EoE and suggest that the loss of DSG1 may potentiate allergic inflammation through the induction of pro-inflammatory mediators such as POSTN.

\section{INTRODUCTION}

Eosinophilic esophagitis (EoE) is a chronic inflammatory disease that has emerged over that last decade on a worldwide scale. ${ }^{1}$ Although symptomatically resembling gastroesophageal reflux disease (GERD), EoE is characterized by immune sensitization to a variety of foods and marked $\mathrm{T}$ helper type 2 (Th2)-associated allergic inflammation in the esophageal mucosa that is largely refractory to acid-suppressive therapy. ${ }^{2}$ During active disease, the histopathological changes within the inflamed esophageal mucosa include the dense accumulation of activated immune cells, including eosinophils, mast cells, and T and B lymphocytes. ${ }^{3}$ Moreover, evidence of dilated intercellular spaces and abnormal epithelial cell proliferation suggest that impaired barrier function (IBF) of the esophageal epithelium may potentially contribute to the pathophysiology of EoE. ${ }^{4-6}$ Although dietary modification (i.e., complete or targeted food antigen avoidance) and swallowed glucocorticoids alleviate much of the disease pathology, EoE still has one of the lowest quality-of-life indexes among other chronic pediatric diseases, including inflammatory bowel disease. ${ }^{7-9}$

Early efforts aimed at the molecular dissection of EoE pathogenesis included gene expression profiling of esophageal mucosal biopsies from patients with active EoE, which identified a striking disease-associated transcript signature that was highly conserved across patients with EoE and largely normalized during glucocorticoid-induced disease remission. ${ }^{10,11}$

\footnotetext{
${ }^{1}$ Divisions of Allergy and Immunology, Cincinnati Children's Hospital Medical Center, University of Cincinnati College of Medicine, Cincinnati, Ohio 45229-3026, USA. ${ }^{2}$ Departments of Medicine, University of North Carolina School of Medicine, Chapel Hill, North Carolina 27599-7545, USA. ${ }^{3}$ Nutrition and Health Department, Nestlé Research Centre, 1000 Lausanne, Switzerland. ${ }^{4}$ Divisions of Pathology and Laboratory Medicine, Cincinnati Children's Hospital Medical Center, University of Cincinnati College of Medicine, Cincinnati, Ohio 45229-3026, USA. ${ }^{5}$ Divisions of Gastroenterology, Hepatology and Nutrition, Department of Pediatrics, Cincinnati Children's Hospital Medical Center, University of Cincinnati College of Medicine, Cincinnati, Ohio 45229-3026, USA and ${ }^{6}$ Departments of Cell and Molecular Physiology, University of North Carolina School of Medicine, Chapel Hill, North Carolina 27599-7545, USA. Correspondence: ME Rothenberg (Rothenberg@cchmc.org) 
Several pro-inflammatory mediators, such as the chemokine (C-C- motif) ligand 26, periostin (POSTN), and tumor necrosis factor, alpha-induced protein 6 , were dramatically elevated in the inflamed esophageal mucosa in $\mathrm{EoE}^{10}$ and in primary esophageal epithelial cells treated with the Th2 cytokine interleukin-13 (IL-13). ${ }^{11}$ A marked reduction in genes involved in epithelial homeostasis was also observed. In particular, the desmosomal cadherin desmoglein-1 (DSG1) exhibited an approximate eightfold decrease in patients with active EoE, which only partially normalized upon disease remission. ${ }^{11}$

DSG1 is an intercellular adhesion molecule belonging to the desmosomal cadherin family, which includes desmogleins (DSG1-4) and desmocollins 1-3. ${ }^{12}$ Desmogleins and desmocollins are involved in maintaining epithelial homeostasis where they display spatially distinct expression patterns at various levels among different stratified epithelia. ${ }^{12,13}$ In particular, DSG1 is highly expressed in the epidermis and localized primarily within the suprabasal epithelial layers, where it regulates cell adhesion and supports epithelial cell differentiation. ${ }^{14}$ In contrast, desmoglein-3 (DSG3) is localized to undifferentiated, basal epithelial cells and has been suggested to promote cell proliferation. ${ }^{15-17}$

Although the function of DSG1 has been well characterized in the epidermis, its role in the esophageal epithelium under both homeostatic and inflammatory conditions remains largely unknown. DSG1 mediates intercellular adhesion by forming calcium-dependent heterotypic and homotypic interactions between adjacent epithelial cells via its amino-terminal ectodomain. ${ }^{18}$ However, it is becoming increasingly appreciated that DSG1 has additional roles beyond that of cell adhesion. ${ }^{19}$ Indeed, DSG1 promotes epithelial differentiation through negatively regulating the activation of extracellular signal-regulated kinase by epidermal growth factor receptor signaling. ${ }^{14}$ DSG1 can also promote epithelial differentiation through its interactions with ERBIN, which sequesters SHOC2 to attenuate RAS-mediated extracellular signal-regulated kinase activation. $^{20}$

In the present study, we sought to determine the functional consequences of DSG1 dysregulation in EoE pathogenesis, as DSG1 was one of the most downregulated genes in the esophageal mucosa of EoE patients. ${ }^{11}$ We hypothesized that the downregulation of DSG1 contributes to the pathological features of the esophageal epithelium in EoE. We demonstrate a marked reduction in the gene and protein levels of DSG1 but not DSG3 in the esophageal mucosa of patients with EoE. Suppression of DSG1 by short hairpin RNA (shRNA) or IL-13 treatment induced IBF in differentiated esophageal epithelial cells grown at the air-liquid interface (ALI); notably, IBF was also observed ex vivo in biopsy samples from patients with EoE. Finally, knockdown of DSG1 was sufficient to induce a gene expression profile, which included POSTN as the top induced gene that substantially overlaps with the transcriptome of the inflamed esophageal mucosa of patients with EoE. These data suggest that the negative regulation of DSG1 by IL-13 can exacerbate inflammation of the esophageal mucosa in EoE by enhancing IBF and initiating a pro-inflammatory gene expression cascade.

\section{RESULTS}

\section{Specific dysregulation of DSG1 in EoE}

We sought to establish the relative levels of all desmoglein genes (DSG1-4) in the esophagus and to determine whether the downregulation of DSG1 was specific among other DSG family members. Whole-transcriptome RNA sequencing of esophageal biopsies from healthy controls (NL; $n=6)$ and patients with active $\operatorname{EoE}(n=10)$ showed a specific and dramatic downregulation of DSG1 in this cohort of patients (Figure 1a). Indeed, DSG1 exhibited a 12.7-fold reduction in patients with active EoE (fragments per kilobase per million mapped reads

a

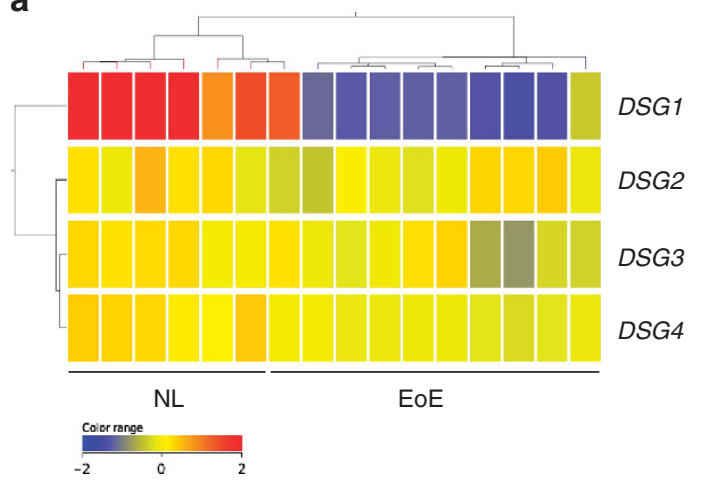

b

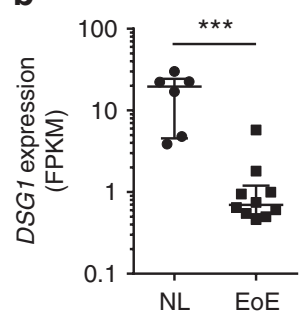

C

d
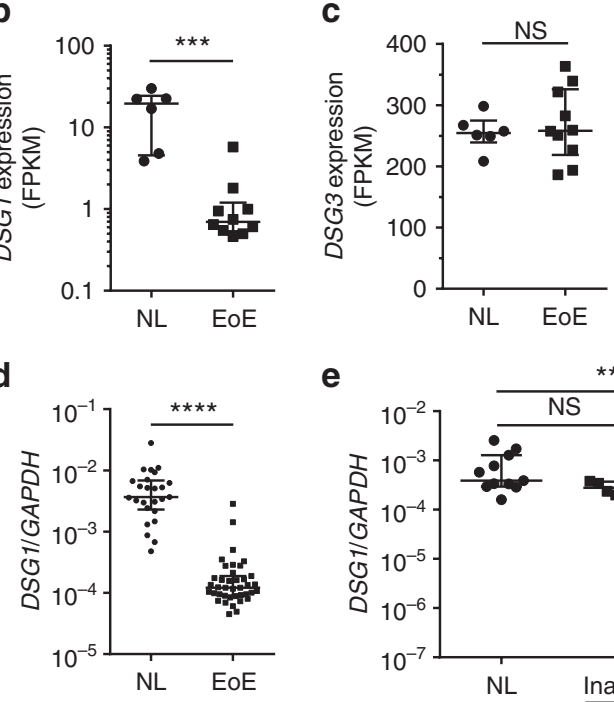

e

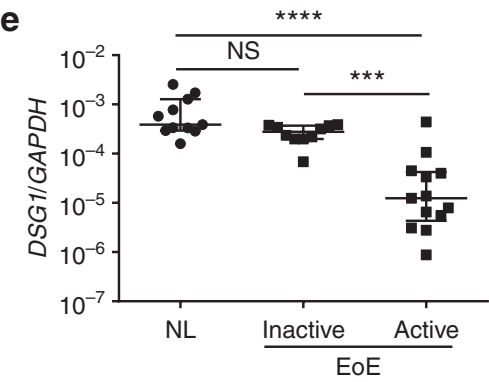

Figure 1 Specific reduction in desmoglein-1 (DSG1) gene expression in eosinophilic esophagitis (EoE). Heatmap depicting expression levels of desmogleins 1-4 (DSG1-4) (a) and individual FPKM values for DSG1 and desmoglein-3 ( $D S G 3 ; \mathbf{b}, \mathbf{c}$ ) from RNA sequencing of esophageal biopsies from 10 patients with active EoE vs. 6 healthy controls (NL). Quantitative PCR (qPCR) analysis of DSG1 expression in esophageal biopsies from $\mathrm{NL}(n=25)$ and patients with active $\operatorname{EoE}(n=39)(\mathrm{d})$. qPCR analysis of $D S G 1$ expression in esophageal biopsies from NL $(n=11)$ and patients with inactive $(n=10)$ or active $(n=13) \operatorname{EoE}(\mathbf{e})$. Data are represented as the median + interquartile range: FPKM, fragments per kilobase per million mapped reads; NS, not significant; ${ }^{\star \star \star} P<5 \times 10^{-4}$; ${ }^{\star \star \star \star} P<1 \times 10^{-4}$. 
$($ median + interquartile range $)=19.6+(4.6-24.4)$ in NL and $0.70+(0.5-1.2)$ in EoE; $\left.P=1 \times 10^{-3}\right)$. Notably, the most abundant DSG expressed in the esophageal mucosa was DSG3, which did not display differential expression in EoE (fragments per kilobase per million mapped reads (median + interquartile range $)=254.4+(239.0-279.5)$ in $\mathrm{NL}$ and $258.2+(218.6-$ 326.1) in EoE; $P=0.75$; Figure 1c). We examined the downregulation of DSG1 in a larger cohort of NL and patients with active EoE disease $(n=25$ and 39, respectively) by quantitative PCR (qPCR) and detected a 22.1-fold reduction $\left(P=1 \times 10^{-4}\right)$ in the esophageal expression of DSG1 in active EoE (Figure 1d). Finally, we assessed esophageal DSG1 levels in patients with inactive $\operatorname{EoE}(n=10)$ following swallowed glucocorticoid therapy (Figure 1e). During disease remission, expression of DSG1 normalized to similar levels that were observed in NL $(n=11)$, yet was significantly different compared to patients with active disease $(n=13$; $\left.P=3 \times 10^{-4}\right)$.

We next performed immunofluorescent and/or immunohistochemical staining for DSG1, DSG3, and E-cadherin in the esophageal mucosa of NL and patients with active EoE to characterize protein expression and localization. Consistent with previous reports, ${ }^{21}$ expression of DSG1 localized to the cell surface and was restricted to the suprabasal esophageal epithelium in NL, whereas DSG1 staining was remarkably absent in patients with active EoE (Figure 2a, upper panel). Conversely, DSG3 was abundantly expressed throughout most of the esophageal epithelium in both NL and EoE, with more concentrated staining within the basal epithelial cell layers (Figure 2a, lower panel). Immunohistochemical staining for DSG1 revealed a similar downregulation in EoE, whereas expression of E-cadherin, a ubiquitously expressed cadherin molecule that regulates epithelial homeostasis and barrier formation, ${ }^{22}$ was unchanged between NL and patients with active EoE (Figure 2b); these findings were supported at the gene level by qPCR analysis of esophageal biopsies from NL and active EoE patients (data not shown). These cumulative data indicate a specific downregulation of the DSG1 gene and protein in EoE, and that this is unique from the expression patterns of other desmogleins and E-cadherin.

\section{Loss of DSG1 regulates esophageal epithelial cell integrity in vitro}

In order to investigate the functional consequences of DSG1 dysregulation on the esophageal epithelium, we developed a modified ALI culture system to induce a stratified esophageal epithelium in vitro. Similar ALI models have been used to study differentiated epithelium of both epidermis and esophagus. ${ }^{23,24}$ Confluent monolayers of immortalized esophageal epithelial cells (EPC2), which have been previously shown to form stratified esophageal epithelium, ${ }^{25-27}$ were exposed to the ALI in the presence of high $\mathrm{Ca}^{2+}(1.8 \mathrm{~mm})$. Following 5-7 days of differentiation at the ALI, the EPC2 cells formed a stratified epithelium as indicated by the differential hematoxylin and eosin staining when compared with submerged cells (Figure 3a). Gene expression analyses after ALI exposure

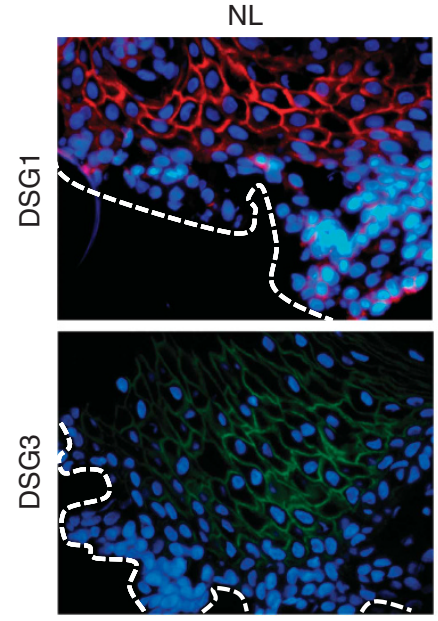

NL
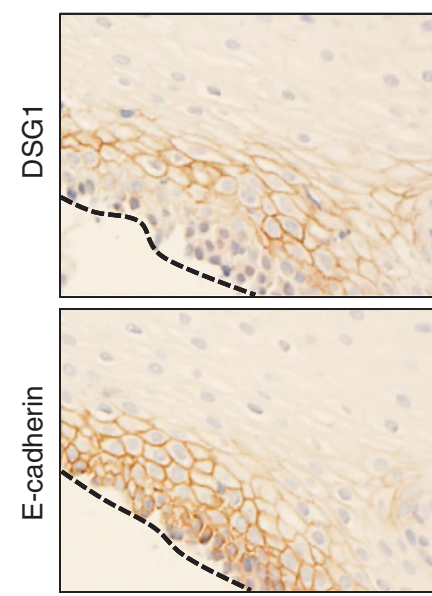

Figure 2 Loss of desmoglein-1 (DSG1) protein expression in eosinophilic esophagitis (EoE). Immunofluorescence (a) or immunohistochemical staining (b) of esophageal biopsy sections from healthy controls (NL) and patients with active EoE. In panel a, DSG1 (upper panel, in red) and desmoglein-3 (DSG3) (lower panel, in green) are shown. Nuclei are stained with 4',6-diamidino-2-phenylindole (blue). In panel b, DSG1 (upper panel, in brown) and E-cadherin (lower panel, in brown) are shown. Dashed lines in panels $\mathbf{a}$ and $\mathbf{b}$ indicate the basal epithelial layer. Images in are representative of four patients per group.

showed significant induction of DSG1 and keratin 10 (411-fold, $P<1 \times 10^{-3}$ and 6,240-fold, $P<1 \times 10^{-2}$, respectively), both of which are expressed specifically in differentiated esophageal epithelial cells ${ }^{21,28,29}$ (Figure 3b,c).

We next utilized lentiviral shRNA gene silencing to directly examine the impact of DSG1 dysregulation on esophageal epithelial cell adhesion. EPC2 cells that were stably transduced with a DSG1 shRNA exhibited a 92\% reduction in DSG1 expression compared with cells transduced with a nonsilencing control (NSC) shRNA $\left(P<5 \times 10^{-3}\right.$; Figure 4a), whereas DSG3 levels remained unaffected (Figure $4 \mathbf{b}$ ). Histological analyses of the NSC shRNA-transduced cells grown at the ALI showed normal stratification with a differentiated, intact esophageal epithelium (Figure 4c, upper panel). However, although the differentiation appeared normal in the DSG1 shRNA-transduced cells, prominent cellular separation 


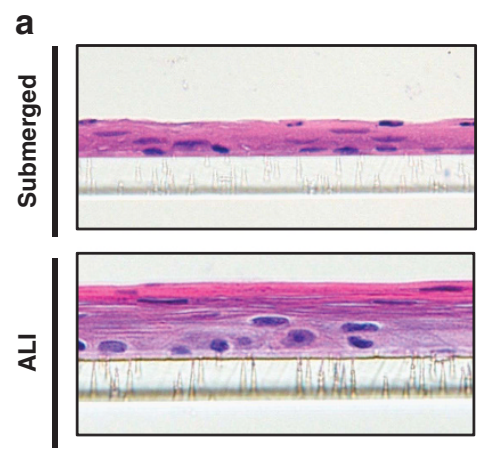

b

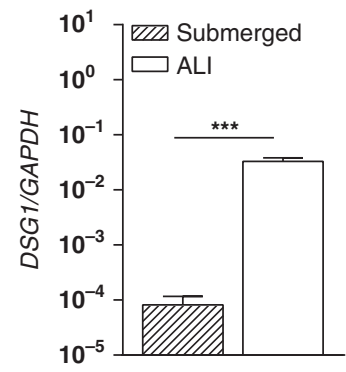

C

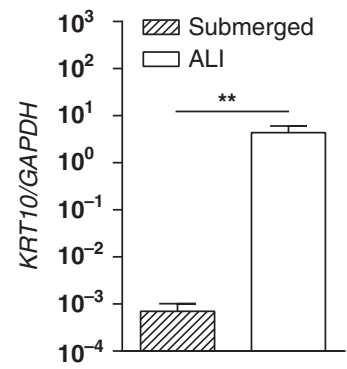

Figure 3 Differentiation of esophageal epithelial cells at the air-liquid interface (ALI). hematoxylin and eosin (H\&E)-stained sections of EPC2 cells grown in submerged cultures or differentiated at the ALI (a). Quantitative PCR (qPCR) analysis of desmoglein-1 (DSG1) (b) and keratin 10 $(K R T 10)(\mathbf{c})$ expression in submerged or ALI-differentiated EPC2 cells. Data are representative of four experiments performed in duplicate and are represented as the mean + s.e.m.: ${ }^{* *} P<5 \times 10^{-3} ;{ }^{* * *} P<5 \times 10^{-4}$.

was evident in the suprabasal layers (arrows, Figure 4c, lower panel). Importantly, this cellular separation was consistently observed only in the DSG1-deficient cells (and not the NSC controls cells) using multiple DSG1 shRNA clones, indicating that the observed phenotype was not because of sample sectioning (data not shown). A similar phenotype was observed when ALI-differentiated EPC2 cells were treated directly with recombinant wild-type exfoliative toxin A (ETA), a DSG1specific protease produced by Staphylococcus aureus and the causative agent of epidermal blistering in staphylococcal scalded-skin syndrome ${ }^{30}$ (Figure 4d, lower panel). Notably, the inactive mutant form of ETA (S195A) did not induce cellular separation (Figure 4d, upper panel). To directly measure whether loss of DSG1 reduced esophageal epithelial cell adhesion, NSC or DSG1 shRNA-transduced cells were subjected to a dispase adhesion assay. Following mechanical disruption, a significantly greater amount of cell dissociation was observed in cells deficient in DSG1 than in control cells $\left(P<5 \times 10^{-2} ;\right.$ Figure 4e,f).

\section{IBF in EoE and in DSG1-deficient esophageal epithelial cells in vitro}

To assess the integrity of the esophageal epithelium in EoE at the ultrastructural level, we performed electron microscopy on NL and EoE patient biopsies. Although the esophageal epithelium of NL patients was composed of cohesive, intact epithelia, prominent dilated intercellular spaces were evident throughout the esophageal epithelium of patients with EoE (arrowheads, Figure 5a). These findings, which have been noted in previous studies of both EoE and GERD, ${ }^{31,32}$ were consistent across multiple sections from the same patient and among different patients. We next assessed whether IBF is indeed a pathological defect in EoE by measuring transepithelial electrical resistance (TER) ex vivo in esophageal biopsies from NL and patients with EoE (Figure 5b). We found that transcellular permeability was reduced by $\sim 62 \%$ in patients with EoE compared with in NL $\left(R_{\mathrm{T}}=84 \pm 28\right.$ vs. $224 \pm$ $23 \mathrm{ohms} \times \mathrm{cm}^{2}$, respectively; $P<5 \times 10^{-3}$ ). We also analyzed paracellular permeability in EoE by measuring macromolecular flux in esophageal biopsies and observed an $\sim 46$-fold increase in those from patients with EoE compared with NL (Figure 5c) $\left(P<5 \times 10^{-2}\right)$. Notably, esophageal expression of occludin $(O C L N)$ and genes in the claudin $(C L D N)$ and tight junction protein $(T J P)$ families was not reduced in EoE, suggesting the IBF in EoE occurs in the absence of marked alteration to tight junctions (Supplementary Figure S1A-C online).

We hypothesized that the cellular separation that occurs within the esophageal epithelium following the loss of DSG1 (Figure 4c) may contribute to the IBF observed in EoE (Figure 5a-c). To test this possibility, we measured TER and paracellular permeability in the ALI-differentiated NSC or DSG1 shRNA-transduced EPC2 cells. Reduced DSG1 expression resulted in impaired TER $\left(P<5 \times 10^{-3}\right.$, Figure 5d) and increased fluorescein isothiocyanate-dextran flux $\left(P<5 \times 10^{-2}\right.$, Figure $5 \mathbf{e}$ and $\mathbf{f}$ ) by $\sim 42 \%$ and $33 \%$, respectively. These data identify a novel mechanism whereby loss of DSG1 negatively affects esophageal epithelial integrity and is sufficient to induce IBF.

\section{IL-13 regulates DSG1 and promotes IBF in differentiated esophageal epithelial cells}

IL-13 is a critical Th2 cytokine capable of eliciting some of the transcriptional changes within the inflamed esophageal epithelium that are associated with EoE, including the downregulation of multiple epithelial cell differentiation genes. ${ }^{11,33}$ Therefore, we assessed the effects of IL-13 on the integrity and barrier formation of ALI-differentiated esophageal epithelial cells. Esophageal epithelial cells were left untreated or treated with 10 or $100 \mathrm{ng} \mathrm{ml}^{-1}$ IL-13 continuously throughout the ALI differentiation process. Both concentrations of IL-13 induced partial cellular separation within the suprabasal epithelial layers (arrows, Figure 6a, middle and lower panels).

As the IL-13-induced suprabasal cell separation partially reflected the phenotype of DSG1-deficient cells (Figure 4c), we next investigated the ability of IL-13 to regulate DSG1 expression. IL-13 treatment suppressed the induction of DSG1 in ALI-differentiated esophageal epithelial cells (Figure 6b), whereas induction of KRT10 (Figure 6c) and DSG3 (data not shown) were unaffected. Importantly, IL-13 


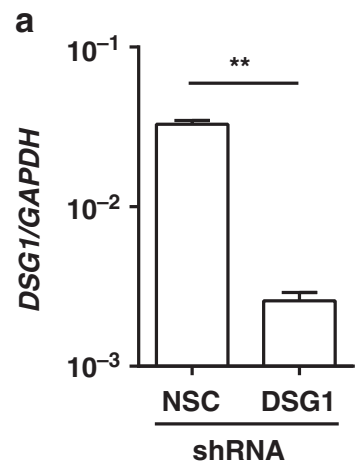

C

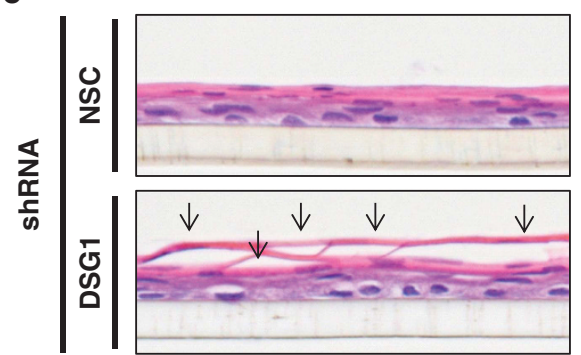

b

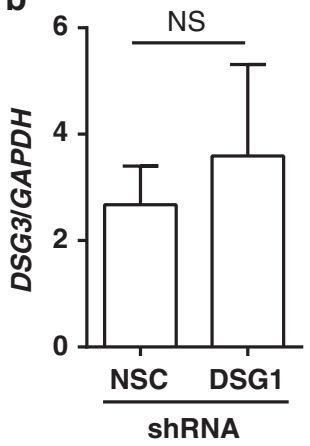

d

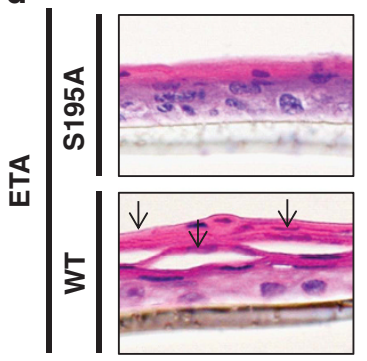

e

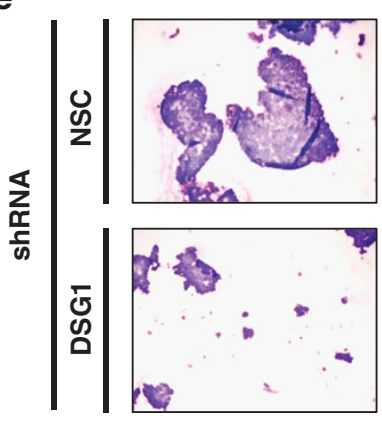

f

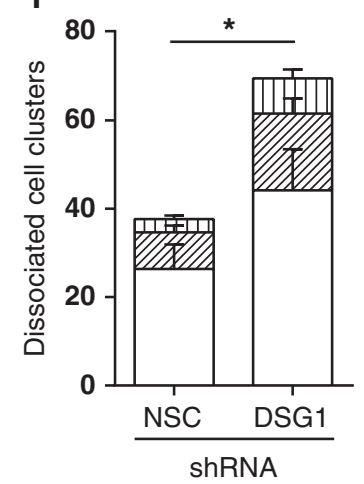

$\square$ Single cells

ZII Two-cell clusters

पा Three-cell clusters

Figure 4 Loss of desmoglein-1 (DSG1) reduces esophageal epithelial cell adhesion. quantitative PCR (qPCR) analysis of DSG1 (a) and desmoglein-3 (DSG3) (b) in air-liquid interface (ALI)-differentiated EPC2 cells stably transduced with non-silencing control (NSC) or DSG1 short hairpin RNA (shRNA). Hematoxylin and eosin (H\&E)-stained sections from stably transduced cells differentiated at the ALI (c). H\&E-stained sections from EPC2 cells exposed to the air interface and treated with $10 \mu \mathrm{g} \mathrm{ml}^{-1}$ exfoliative toxin A (ETA; wild-type (WT)) or the S195A inactive mutant for $24 \mathrm{~h}$ (d). Arrows (c,d) indicate cell separation within the suprabasal epithelium. Cytospins from NSC or DSG1 shRNA-transduced EPC2 cells following dispase adhesion assays (e) and quantification of dissociated cell clusters are shown (f). Images in panels c-e are representative of four to five experiments performed in duplicate. Data in panels $\mathbf{a}, \mathbf{b}$ and $\mathbf{e}$ are from three experiments performed in duplicate and are represented as the mean + s.e.m.: NS, not significant; ${ }^{*} P<5 \times 10^{-2} ;{ }^{\star *} P<5 \times 10^{-3}$.

also promoted IBF, as a significant reduction in TER was observed at both 3 and 5 days after treatment with IL-13 (100 $\mathrm{ng} \mathrm{ml}^{-1}$ ) compared with untreated cells (Figure $\mathbf{6 d}$ ).

We also tested whether IL-13 could attenuate desmoglein-1 expression in vivo using a transgenic murine model of EoE. Doxycycline-inducible expression of IL-13 in the lung (using the Clara cell-specific promoter $\mathrm{CC} 10$ ) has been shown to induce an EoE phenotype in mice. ${ }^{34}$ Elevated IL-13 levels in the bronchoalveolar lavage fluid and induced esophageal eosinophilia were detected in the doxycycline-treated animals (Supplementary Figure S1A, B). Overexpression of IL-13 in treated (+ doxycycline) mice reduced DSG1 messenger RNA and protein levels in the esophageal mucosa as compared with untreated ( - doxycycline) mice (Supplementary Figure S1C-E).

\section{Loss of DSG1 primes for the innate inflammatory transcript signature}

DSG1 has been shown to counter-regulate epidermal growth factor receptor signaling, and more recently RAS-mediated signaling, through mechanisms that are independent of its fulllength, adhesive amino-terminal domain. ${ }^{14,20}$ Therefore, we hypothesized that DSG1 deficiency may also regulate downstream signaling responses associated with the Th2 inflammation in EoE. To test this possibility, we performed microarray analyses on NSC and DSG1 shRNA-transduced EPC2 cells following ALI differentiation. Interestingly, 63 transcripts coding for 53 unique genes were differentially expressed in the DSG1-deficient cells compared to control cells $\left(P<5 \times 10^{-2}\right.$, fold change $>2.0$; Figure 7a). Notably, there 

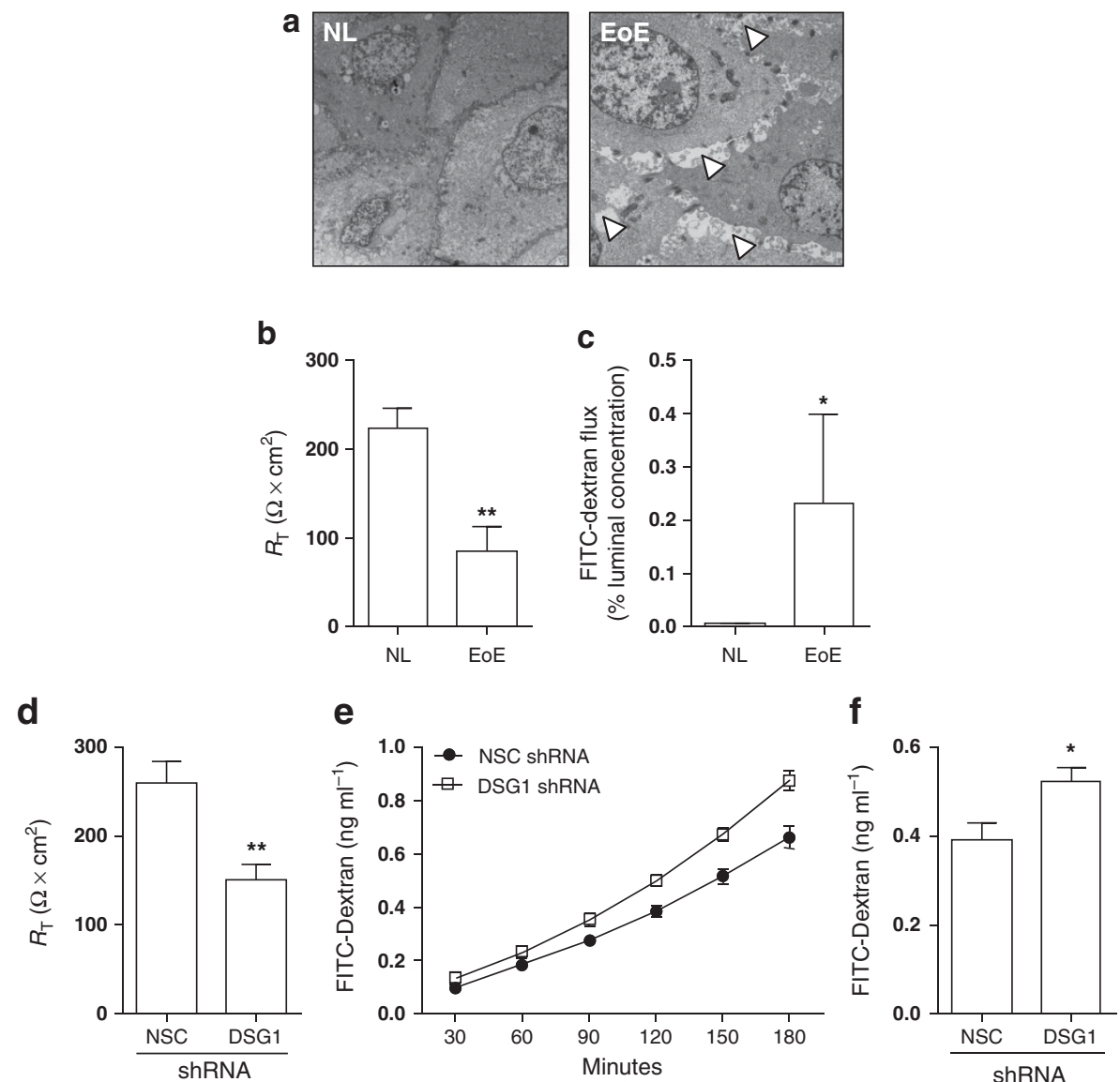

Figure 5 Impaired barrier function (IBF) in eosinophilic esophagitis (EoE) can be replicated in DSG1-deficient esophageal epithelial cells. Representative electron micrographs of esophageal biopsies from healthy controls $(\mathrm{NL})(n=3)$ and patients with active EoE ( $n=3)$. Arrowheads indicate the presence of dilated intercellular spaces (DIS) in EoE (a). Transepithelial electrical resistance (TER; $\left.R_{\mathrm{T}}\right)$ measurements from esophageal biopsies from NL and patients with active EoE ( $n=6$ and 9 , respectively; $\mathbf{b})$. Fluorescein isothiocyanate (FITC)-dextran flux assays from NL and active EoE esophageal biopsies ( $n=6$ and 4, respectively; $\mathbf{c})$. TER $\left(R_{\mathrm{T}}\right)$ measurements from non-silencing control (NSC) and DSG1 short hairpin RNA (shRNA)-transduced EPC2 cells following air-liquid interface (ALI) differentiation (d). Kinetic analysis of FITC-dextran flux was also performed (e). Total FITC-dextran flux following $180 \mathrm{~min}$ are depicted in panel f. Data in panels $\mathbf{d}-\mathbf{f}$ are from two independent experiments performed in quadruplicate. All data are represented as the mean + s.e.m.: ${ }^{\star} P<5 \times 10^{-2}$; ${ }^{\star \star} P<5 \times 10^{-3}$.

was a substantial overlap between this transcript profile and the EoE transcriptome identified in patient esophageal biopsies by RNA sequencing; $60 \%$ of the unique DSG1-regulated transcripts were also dysregulated in esophageal biopsies of patients with EoE (Figure $7 \mathbf{b}$ and Supplementary Table S1 online). In particular, $P O S T N$ was the most highly induced gene upon DSG1 knockdown in esophageal epithelial cells (2.5-fold) and that was dramatically dysregulated in esophageal tissue of patients with EoE (384-fold induction; Supplementary Table S1).

We next validated the significant increase in POSTN levels in DSG1-deficient esophageal epithelial cells by qPCR and observed a 17 -fold increase in POSTN in the DSG1 vs. NSC shRNA-transduced cells $\left(P<5 \times 10^{-2}\right.$; Figure 8a). Further, qPCR analysis demonstrated a significant increase in POSTN expression in the esophageal mucosa of patients with active EoE compared with NL $\left(P<1 \times 10^{-4}\right.$, fold change $=2,047$; Figure 8b). Notably, POSTN levels in EoE showed a significant inverse correlation with DSG1 expression (same cohort as in Figure 1d; Spearman's $r=-0.33, P=0.02$; Figure 8c).
Together, these data indicate that IL-13-induced loss of DSG1 augments the allergic inflammatory response by promoting IBF and likely elevates pro-inflammatory gene expression in EoE (Figure 9).

\section{DISCUSSION}

The data presented herein characterize the pathological impact of DSG1 dysregulation in EoE and define a nonredundant role for DSG1 in the regulation of the esophageal epithelial barrier function and homeostasis. In particular, we demonstrated a specific and marked downregulation of DSG1 in the esophageal epithelium of patients with EoE that was largely reversible with patient disease status. This loss in DSG1 expression was sufficient to induce cell separation and IBF in the esophageal epithelium. Moreover, DSG1 deficiency led to an esophageal epithelial transcript signature that included increased gene expression of the pro-inflammatory extracellular matrix molecule POSTN. These findings are particularly notable in view of the relatively high abundance of DSG3 expression in 


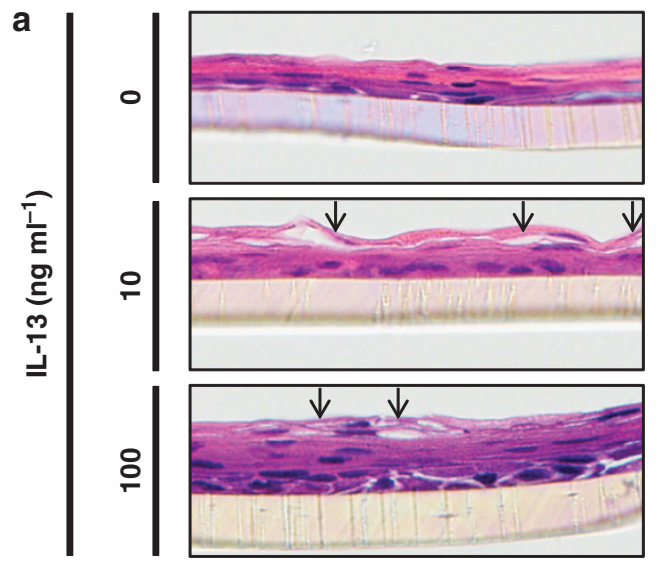

b

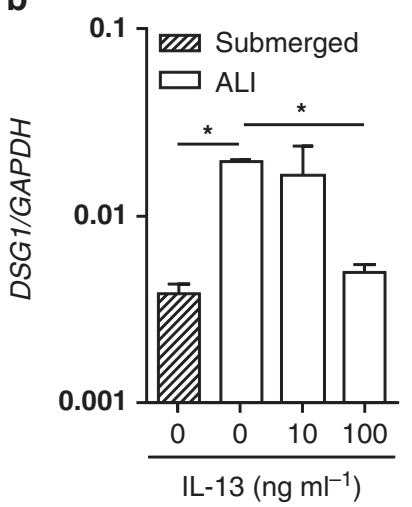

C

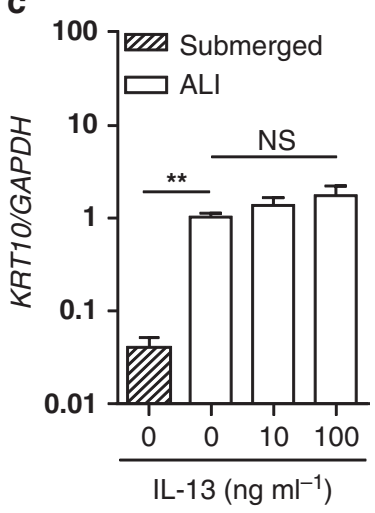

d

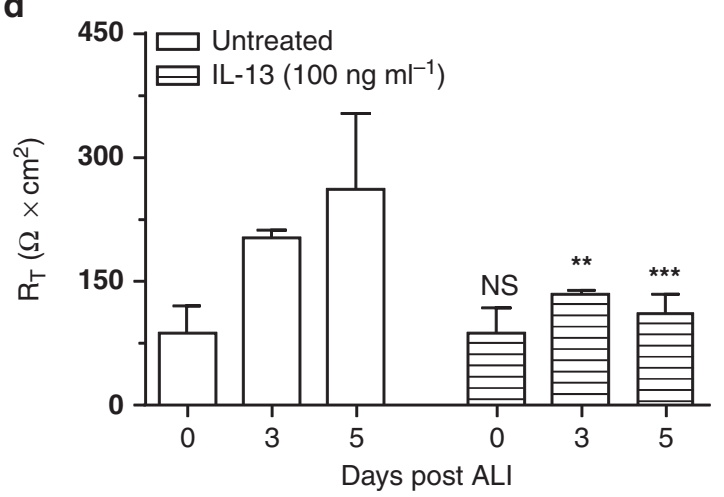

Figure 6 Interleukin-13 (IL-13) downregulates DSG1 and promotes impaired barrier function (IBF) in esophageal epithelial cells. Hematoxylin and eosin (H\&E)-stained sections of EPC2 cells differentiated at the air-liquid interface (ALI) in the presence of 0 (untreated), 10 , or $100 \mathrm{ng} \mathrm{ml}^{-1}$ IL-13 (a). Arrows indicate a cell separation within to the suprabasal epithelium. Images are representative of three experiments performed in duplicate. Expression levels of DSG1 (b) and keratin 10 (KRT10) (c) were measured by quantitative PCR (qPCR) in submerged or ALI-differentiated EPC2 cells in the absence ( $0 \mathrm{ng} \mathrm{ml}^{-1}$ ) or presence of IL-13 (10 or $100 \mathrm{ng} \mathrm{ml}^{-1}$ ). Transepithelial electrical resistance (TER; $R_{\mathrm{T}}$ ) measurements on EPC2 cells at 0,3 , and 5 days following differentiation at the ALI in the absence (untreated) or presence of IL-13 $\left(100 \mathrm{ng} \mathrm{ml}^{-1}\right)$ (d). Data are from three experiments performed in duplicate and are represented as the mean + s.e.m.: NS, not significant; ${ }^{* \star} P<5 \times 10^{-3}$; ${ }^{* \star \star} P<1 \times 10^{-4}$ as compared with the untreated cells at the same days after ALI differentiation.

EoE and in our in vitro model, demonstrating the lack of redundancy for DSG1's regulation of esophageal responses, particularly during allergic inflammation. Interestingly, in
GERD in which dilated intercellular spaces and IBF have been attributed to acid exposure of the esophageal epithelium, esophageal expression of DSG1 (and DSG3 to a lesser extent) is increased, ${ }^{35}$ further suggesting a unique, nonredundant and essential role for DSG1 in regulating disease processes specifically linked with EoE pathology.

Our data identify IL-13 as a potent regulator of DSG1 expression in human esophageal epithelial cells cultured at the ALI and in mouse esophageal epithelial cells in a murine model of EoE. This latter finding is quite striking, given the physiological differences between the human and mouse esophagus (not keratinized vs. keratinized, respectively) and the existence of multiple murine $D s g 1$ isoforms $(\alpha, \beta$, and $\gamma),{ }^{36}$ which were universally detected in our qPCR analysis and immunofluorescent staining (see Supplementary Materials and Methods). Previous work has demonstrated a pronounced effect of IL-13 on global gene expression in primary esophageal epithelial cells and, in particular, genes involved in epithelial differentiation. ${ }^{11,33}$ DSG1 was not shown to be regulated by IL-13 in these data, which, on the basis of our data in the ALI system (Figure 3), we hypothesize was due to the low levels of DSG1 expression in esophageal epithelial cells grown in the submerged culture conditions used in these studies. Interestingly, in skin keratinocytes, which express high baseline levels of DSG1 even in submerged cultures, IL-4 has been shown to downregulate DSG1 expression in vitro. ${ }^{37}$ Notably, both IL-13 and IL-4 are significantly increased in the peripheral blood and esophageal mucosa of patients with active $\mathrm{EoE},{ }^{38,39}$ and preliminary data indicate that IL- 4 is also capable of negatively regulating DSG1 expression and inducing IBF in ALI-differentiated esophageal epithelial cells in vitro (data not shown). These data, together with our findings that esophageal expression of DSG1 normalizes in EoE patients during disease remission, indicate DSG1 is negatively regulated by Th2 cytokines during allergic inflammation.

Within the epithelial barrier of the epidermis and intestine, the selective ability to discriminate the uptake of molecules based on size and charge is attributed to the tight junction complex. $^{40}$ For example, several tight junction genes are expressed within the epidermis including CLDN1, CLDN4, TJP1, and OCLN. ${ }^{41}$ While CLDN1 has been demonstrated to have an essential role in epidermal barrier function in vivo, ${ }^{42}$ a recent study has shown CLDN4, TJP1, and OCLN can also independently regulate epidermal permeability in vitro. ${ }^{43}$ Although the esophageal epithelial barrier has been studied primarily in the context of GERD, TJPs have been shown to have no correlation with GERD pathogenesis. ${ }^{44}$ Moreover, our data (Supplementary Figure S1) demonstrate no decrease in genes encoding CLDNs, TJPs, or OCLN in EoE; none of these genes were dramatically altered in DSG1-deficient cells following shRNA transduction or IL-13 treatment (Supplementary Table S3). Together, these data suggest physiologically distinct mechanisms regulate the epithelial barrier properties of the esophagus and the epidermis.

Despite our evidence substantiating IBF as a bona fide pathological feature in EoE, little is known regarding the role of 
a

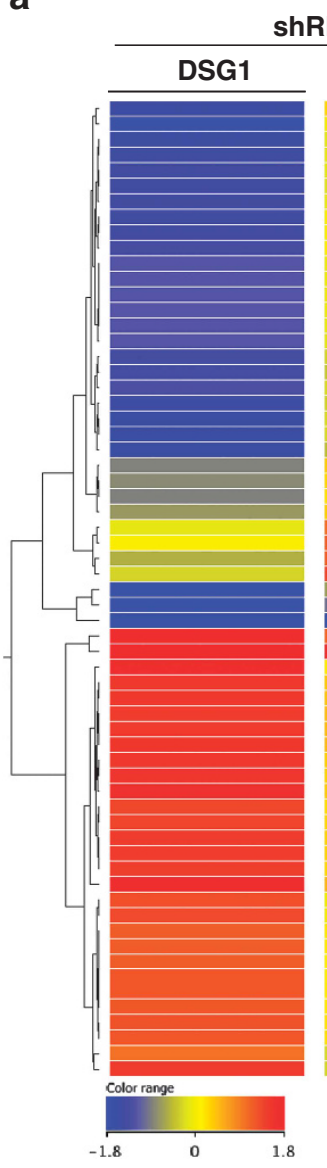

b

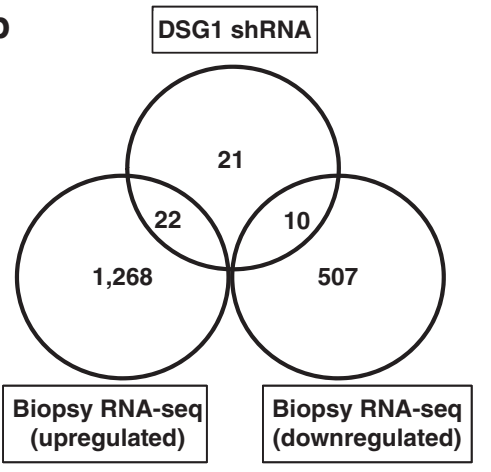

Figure 7 Loss of DSG1 promotes epithelial pro-inflammatory transcriptional responses. Heatmap of 63 transcripts with differential expression $\left(P<5 \times 10^{-2}\right.$, fold change $\left.>2.0\right)$ in DSG1-deficient EPC2 cells compared with non-silencing control (NSC) cells following air-liquid interface (ALI) differentiation (a). Venn diagram depicting the number of genes $(n=32$; boxed transcripts in panel a) dysregulated following DSG1 knockdown that overlap with differentially expressed genes in esophageal mucosa of eosinophilic esophagitis (EoE) patients $(n=10)$ compared with healthy control (NL) patients $(n=6)$ identified by RNA sequencing (b).

the esophageal barrier in regulating allergic inflammation. Within the epidermis, Langerhans cells continually sample external antigens by inducing transient reorganization of tight junctions within the stratum corneum without affecting barrier integrity; this homeostatic surveillance is further amplified in activated Langerhans cells. ${ }^{45}$ These data suggest a potential mechanism by which IBF could lead to increased antigen sensitization, which is dependent on tissue-resident antigen presenting cells. It is important to note that Langerhans cells ${ }^{46,47}$ and high baseline expression of the high-affinity $\operatorname{IgE}$ receptor FceRI (ref. 48) have been observed in both healthy and inflamed esophageal epithelium. Moreover, data suggesting esophageal epithelial cells can present antigen and major histocompatibility complex class II expression is increased in EoE (ref. 49) further suggesting that the esophagus is not a unifunctional, static organ involved solely in food transport but rather has an active role in immunosurveillance during allergic inflammation.

Our finding of elevated POSTN expression in DSG1-deficient cells is particularly notable as periostin has been implicated in multiple allergic inflammatory disease including asthma, atopic dermatitis, and EoE. ${ }^{50-52}$ periostin can directly enhance eosinophil adhesion, ${ }^{50,53}$ as well as increase keratinocyte production of TSLP (thymic stromal lymphopoetin), ${ }^{52}$ a potent Th2-skewing cytokine that has been genetically linked to EoE susceptibility. ${ }^{54,55}$ Although periostin is expressed primarily in fibroblasts, treatment of both bronchial epithelial cells ${ }^{56}$ and esophageal epithelial cells ${ }^{50}$ with IL-13 induces POSTN expression in these cell types. Interestingly, periostin has been shown to enhance signaling through epidermal growth factor receptor and integrin $\alpha_{v} \beta_{5}$ to induce epithelial-mesenchymal transition, ${ }^{57}$ a process associated with the loss of epithelial cell markers as epithelial cells adopt a fibroblast-like phenotype and increased migratory properties (e.g., loss of cell adhesion). ${ }^{58}$ Although a previous report demonstrated that DSG1 supports epithelial differentiation and reduces proliferative capacity through negative regulation of epidermal growth factor receptor signaling, ${ }^{14}$ a similar suppressive effect of DSG1 on epithelial-mesenchymal transition induction has not been addressed. As it has been recently proposed that epithelial-mesenchymal transition is actively occurring in $\mathrm{EoE},{ }^{59,60}$ perhaps the loss of DSG1-dependent adhesion and the upregulation of POSTN synergistically disrupt 
a

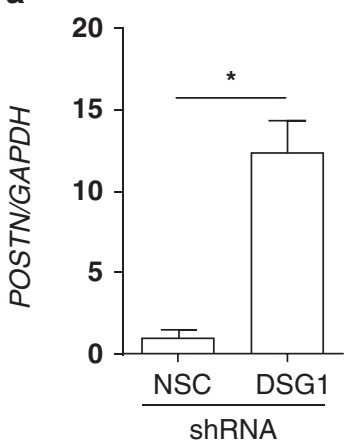

b
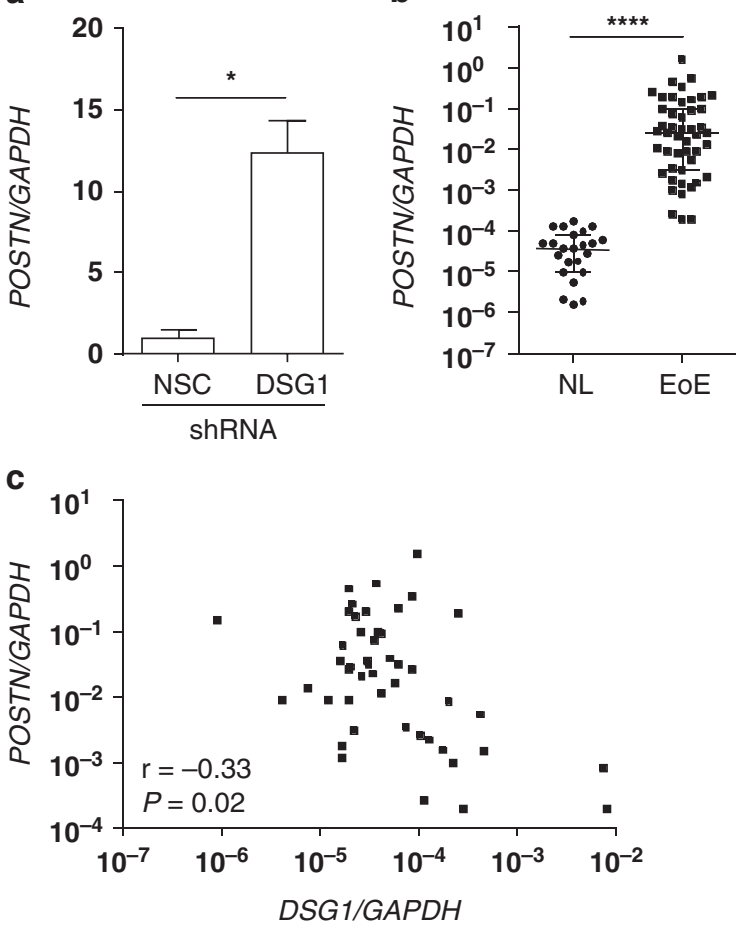

Figure 8 DSG1 deficiency increases periostin (POSTM) expression. Quantitative PCR (qPCR) analysis of POSTN expression in non-silencing control (NSC) and DSG1 short hairpin RNA (shRNA)-transduced EPC2 cells following air-liquid interface (ALI) differentiation (a) and in patient biopsies (same cohort as in Figure 1d) (b). Spearman's correlation between esophageal expression of POSTNand DSG1 (from Figure 1d) in patients with active eosinophilic esophagitis (EoE; $\mathbf{c})$. Data in panel a are representative of three independent experiments performed in duplicate and represented as the mean + s.e.m.: ${ }^{\star} P<5 \times 10^{-2}$; ${ }^{\star \star \star \star} P<1 \times 10^{-4}$.

the homeostatic interactions between epithelial cells and fibroblasts during allergic inflammation.

The clinical importance of DSG1 and its role in maintaining epithelial integrity has been reported in several cutaneous diseases of autoimmune, infectious, and genetic origin. ${ }^{30}$ In pemphigus foliaceous, autoantibodies against the ectodomain of DSG1 induce severe epidermal acantholysis; ${ }^{30}$ interestingly, heightened Th2 inflammation, including elevated Th2 cytokine expression $^{61}$ and the presence of activated eosinophilic infiltrates, ${ }^{62}$ are observed in some instances. In Netherton syndrome, an epidermal inflammatory disease involving IBF with marked eosinophilia and elevated IgE levels, ${ }^{63}$ the primary etiology has been attributed to the genetic loss of the epithelialderived serine protease inhibitor, Kazal type, 5 (SPINK5). ${ }^{64}$ In this disease, the uncontrolled activity of endogenous trypsinlike proteases leads to aberrant cleavage of epithelial barrier proteins within the stratum corneum (including DSG1). Mice deficient in Spink5 display increased DSG1 degradation and significant epidermal pathology, including epidermal acantholysis and IBF. ${ }^{65}$ However, it has been unclear whether the loss of DSG1 alone is sufficient to induce IBF as several proteases (e.g., kallikriens 5, 7, and 14 and elastase 2) and stratum corneum proteins are involved in barrier formation (e.g., filaggrin,

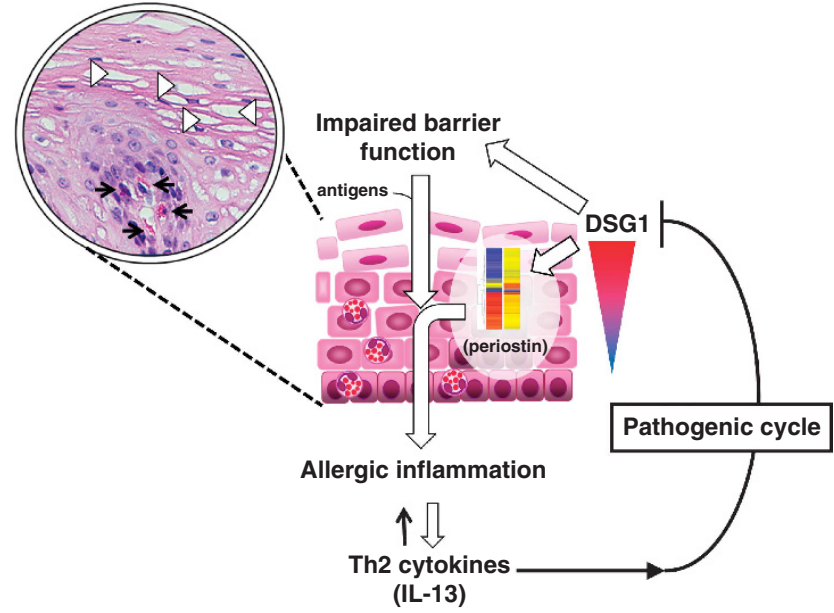

Figure 9 Model of DSG1 dysregulation in eosinophilic esophagitis (EoE) pathogenesis. Downregulation of DSG1 by select T helper type 2 (Th2) cytokines (e.g., interleukin-13) results in impaired barrier function (IBF) and increased antigen exposure as well as the expression of pro-allergic mediators including periostin (POSTM), forming a pathogenic cycle to further exacerbate allergic inflammation. Inset shows hematoxylin and eosin (H\&E) staining of inflamed esophageal mucosa with dilated intercellular spaces (arrowheads) and eosinophilic infiltration (arrows).

involucrin, and loricrin) and are also altered in Netherton syndrome. $^{65-67}$

Recently, genetic variants in DSG1 were identified in a new clinical syndrome manifesting with severe atopic dermatitis, multiple allergies, and metabolic wasting syndrome in two consanguineous families. ${ }^{68}$ Homozygous loss-of-function variants led to reduced expression of DSG1 and loss of cell adhesion. ${ }^{68}$ Notably, one of three patients with severe atopic dermatitis multiple allergies, and metabolic wasting syndrome was reported to have EoE as well as elevated keratinocyte expression of IL5 and TSLP. ${ }^{68}$ Interestingly, in a genome-wide association study, we identified a noncoding variant in intron 1 of DSG1 with suggestive association with EoE risk (meta $\left.P=6.57 \times 10^{-6}\right),{ }^{54}$ the function of which has yet to be determined. Nonetheless, these recent findings coincide with our data, as they provide additional evidence that links DSG1 deficiency to IBF in allergic inflammation.

In summary, the observations presented herein support a pathophysiological role for DSG1 dysregulation in EoE. We propose a pathogenic cycle in which the localized increase in select Th2 cytokines (e.g., IL-13) within the inflamed esophagus decreases expression of DSG1, which is sufficient to weaken esophageal epithelial integrity and induce IBF. This loss of DSG1 initiates a pro-allergic transcriptional response (e.g., increased POSTN expression) that potentiates the inflammatory response and may have far-reaching implications beyond the initial IBF insult, such as promoting epithelialmesenchymal transition (Figure 9).

\section{METHODS}

Human subjects. NL (healthy control patients) were defined as having no history of EoE diagnosis with 0 eosinophils per high-power field 
(HPF) and no evidence of esophagitis within distal esophageal biopsies obtained during the same endoscopy procedure as the analyzed samples. Patients with EoE had clinician-diagnosed EoE and as having active disease in concomitant distal esophageal biopsies with greater than 15 eosinophils per HPF. EoE patients in disease remission (inactive) had clinician-diagnosed EoE and distal esophageal biopsies with less than 15 eosinophils per HPF (range =0-1) following swallowed glucocorticoid therapy.

RNA sequencing and bioinformatic analyses. RNA isolated from esophageal biopsies of $6 \mathrm{NL}$ and 10 patients with active EoE (mean eosinophils per $\mathrm{HPF}=164 \pm 29$ s.e.m.) was subjected to RNA sequencing at the Cincinnati Children's Hospital Medical Center (CCHMC) Gene Discovery and Genetic Variation Core as previously described. ${ }^{69}$ The paired-end sequencing reads were aligned against the GRCh37 genome model using TopHat 2.04 with Bowtie $2.03 .{ }^{70,71}$ The separate alignments were then merged using Cuffmerge with RefSeq gene models as a reference. The aligned reads were then quantified for differential expression analysis using Cuffdiff. ${ }^{72}$ Statistical significance was determined using a $t$-test with a threshold of $P<5 \times 10^{-2}$ and a twofold cutoff filter in GeneSpring 11.5 (Agilent Technologies Incorporated, Clara, CA).

qPCR analysis. RNA samples were prepared as previously described. ${ }^{34}$ Briefly, total RNA (250-500 ng) was DNAase treated, and complementary DNA was generated using the iScript complementary DNA synthesis kit (Bio-Rad Laboratories, Hercules, CA). qPCR was performed, and SYBR Green incorporation was analyzed using iQ5 software (Bio-Rad Laboratories). Specific primer sequences are listed in Supplementary Table S2.

Immunohistochemical and immunofluorescent staining. For immunohistochemical staining, formalin-fixed, paraffin-embedded distal esophageal biopsies were serially sectioned and de-paraffinized using xylene followed by graded ethanol washes. Heat-induced epitope retrieval in sodium citrate buffer $(10 \mathrm{~mm}$ sodium citrate, $0.05 \%$ Tween $20, \mathrm{pH} 6.0$ ) was used, and endogenous peroxidase activity was quenched in $2 \% \mathrm{H}_{2} \mathrm{O}_{2}$. Slides were blocked in phosphatebuffered saline with $3 \%$ goat serum for $1 \mathrm{~h}$ followed by overnight incubation at $4{ }^{\circ} \mathrm{C}$ in the following primary antibodies $\left(2 \mu \mathrm{g} \mathrm{ml}^{-1}\right)$ : anti-DSG1 (sc-2011; Santa Cruz Biotechnology, Santa Cruz, CA), or anti-E-cadherin (number 3195, Cell Signaling Technology, Inc., Danvers, MA). Slides were then washed, incubated for $1 \mathrm{~h}$ at room temperature in biotin-conjugated anti-rabbit $\operatorname{IgG}(1: 250)$, and developed using the Vectastain ABC System according to the manufacturer's protocol (Vector Laboratories, Burlingame, CA). Finally, developed slides were counterstained with Harris hematoxylin. Immunofluorescent staining was performed as previously described ${ }^{50}$ using the following primary antibodies $\left(2 \mu \mathrm{g} \mathrm{ml}^{-1}\right)$ : anti-DSG1 (sc-2011) or anti-DSG3 (sc-23912; Santa Cruz Biotechnology). Nuclei were stained with 4',6-diamidino-2-phenylindole. Patients with active EoE that were analyzed by staining had eosinophil levels ranging from 98 to 265 eosinophils per HPF.

ALI culture system. The esophageal epithelial cell line (human telomerase reverse transcriptase-immortalized EPC2 cell line) was a kind gift from Dr Anil Rustgi (University of Pennsylvania, Philadelphia, PA) and has been extensively characterized in previous studies. ${ }^{25,26,73}$ For the ALI culture system, EPC2 cells were grown to confluence while fully submerged in low-calcium $\left(\left[\mathrm{Ca}^{2+}\right]=0.09 \mu \mathrm{M}\right)$ keratinocyte serum-free media (Life Technologies, Grand Island, NY) on $0.4-\mu \mathrm{m}$ pore-size permeable supports (Corning Incorporated, Corning, NY). Confluent monolayers were then switched to high-calcium $\left(\left[\mathrm{Ca}^{2+}\right]=1.8 \mu \mathrm{M}\right)$ keratinocyte serum-free media for an additional 3-5 days. To induce epithelial stratification and differentiation, the culture medium was removed from the inner chamber of the permeable support in order to expose the cell monolayer to the air interface.
Differentiated esophageal epithelial equivalents were analyzed 5-7 days after exposure.

DSG1 knockdown. EPC2 cells were transduced with shRNA targeting the last exon of DSG1 or a NSC shRNA using the GIPZ lentiviral system (Thermo Fisher Scientific, Rockford, IL). Lentiviral particles were prepared at the CCHMC Viral Vector Core facility. Forty-eight hours after transduction, cells were selected for stable integration using puromycin $\left(1 \mu \mathrm{g} \mathrm{ml}^{-1}\right)$, which was maintained throughout all subsequent experiments. Transduction efficiency was assessed by GFP fluorescence, and knockdown efficiency as compared with NSC shRNA-transduced cells was assessed by qPCR and immunofluorescence staining as described herein.

Expression and purification of recombinant ETA. The plasmids encoding WT ETA or the inactive ETA mutant $S 195 \mathrm{~A}^{74}$ with a $5 \times$ histidine epitope $\operatorname{tag}^{75}$ were kindly provided by Dr John Stanley (University of Pennsylvania, Philadelphia, PA). The complementary DNAs were subcloned into the pT7-7 vector, validated by Sanger sequencing, and purified from isopropyl $\beta$-D-1-thiogalactopyranoside-induced BL21 (DE3) pLysS Escherichia coli cell lysates using $\mathrm{Ni}^{+}$NTA agarose column chromatography (QIAGEN Incorporated, Germantown, MD). Purified ETA WT and S195A proteins were analyzed by SDS-polyacrylamide gel electrophoresis and Coomassie staining or western blot using anti-histidine antibodies; in transiently transfected HEK293T cells, cleavage of DSG1 by WT ETA protein and non-cleavage of DSG1 by S195A ETA protein were confirmed by western blot (data not shown). Final protein concentrations were determined by bicinchoninic acid assay (Thermo Fisher Scientific).

Dispase adhesion assays. Dispase adhesion assays were performed as previously described. ${ }^{76}$ Briefly, confluent monolayers of NSC or DSG1 shRNA-transduced EPC2 cells were grown in $1.8 \mathrm{mM} \mathrm{Ca}^{2+}$ for $24 \mathrm{~h}$, washed twice with phosphate-buffered saline, and then detached from tissue culture wells by incubation in $2.4 \mathrm{U} \mathrm{ml}^{-1}$ dispase (Life Technologies Corporation) for $15-20 \mathrm{~min}$ at $37^{\circ} \mathrm{C}$. Detached monolayers were then subjected to mechanical stress by pipetting with a 1-ml pipet five times. Aliquots were then cyto-centrifuged and stained with Hema 3 (Thermo Fisher Scientific). One-, two-, and three-cell clusters were counted from the entire field under $\times 10$ magnification.

Microarray analyses. RNA was isolated using the miRNeasy kit (QIAGEN Incorporated) according to the manufacturer's protocol. RNA quality assessment, library preparation, hybridization to the GeneChip Human Gene 2.0 ST exon array (Affymetrix, Santa Clara, $\mathrm{CA}$ ), and analysis were performed at the CCHMC Gene Expression Microarray Core. Expression profiles were analyzed using GeneSpring 11.5 (Agilent Technologies Incorporated), and statistical significance was determined using a $t$-test with a threshold of $P<5 \times 10^{-2}$ and a twofold cutoff filter.

Electron microscopy. Transmission electron microscopy was performed at the Pathology Research Core at CCHMC. Biopsy specimens used in this analysis were from NL $(n=3)$ and from patients with active $\operatorname{EoE}(n=3)$ who had a previous EoE diagnosis and an esophageal biopsy with greater than 15 eosinophils per HPF (range= 60-100 eosinophils per HPF). The representative images shown were taken at $\times 10,000$ magnification.

TER and paracellular flux assays. For ex vivo studies, esophageal biopsies from NL or patients with active EoE were mounted into miniUssing chambers, and TER measurements and paracellular flux assays using fluorescein isothiocyanate-dextran (average molecular weight $=4 \mathrm{kDa}$ ) were performed as previously described. ${ }^{77}$ A total of six NL biopsies were analyzed for TER and paracellular permeability. A total of nine and four biopsies from patients with EoE were assessed for TER and paracellular permeability, respectively. To account for potential differences in biopsy thickness across the different patient 
groups, an arbitrary cutoff of $2 \%$ fluorescein isothiocyanate-dextran flux was used, resulting in five of the nine biopsies from patients with EoE being excluded. The data shown represent the mean \pm s.e.m. in vitro measurements for TER following IL-13 treatment were assessed using an EVOM (World Precision Instruments, Inc., Sarasota, FL), whereas TER and paracellular flux assays in DSG1-deficient cells were performed as previously described. ${ }^{78}$

Statistical analyses. Statistical significance was determined using a $t$ test (two-tailed). Non-normally distributed data from patient biopsy samples were analyzed using a Mann-Whitney test, and the Spearman correlation was used to test for correlated gene expression. All statistical analyses were performed using GraphPad Prism (GraphPad Software Incorporated, La Jolla, CA).

Study approvals. For human subjects, written informed consent was obtained before a patient's enrollment in the studies, and all human studies were approved by the CCHMC Institutional Review Board (IRB protocol 2008-0090). All experiments involving mice were approved by the CCHMC IACUC.

SUPPLEMENTARY MATERIAL is linked to the online version of the paper at http://www.nature.com/mi

\section{ACKNOWLEDGMENTS}

This work was supported in part by NIH U19 AI070235, NIH R01 DK076893, the PHS Grant P30 DK0789392, Food Allergy Research \& Education (formerly Food Allergy Project/Food Allergy Initiative) and Food Allergy \& Anaphylaxis Network), the Buckeye Foundation, the Campaign Urging Research for Eosinophilic Diseases (CURED) Foundation, and the Angels for Eosinophilic Research Foundation. JDS was supported by a T32 NIH training Grant (HL091805) and the Thrasher Research Fund Early Career Award (NR-0171). We thank Dr John Stanley (University of Pennsylvania) for the ETA constructs and Dr Anil Rustgi (University of Pennsylvania) for the EPC2-human telomerase reverse transcriptase cell line. We also thank Shawna Hottinger for editorial assistance, all of the participating families and the Cincinnati Center for Eosinophilic Disorders, and members of the Division of Allergy and Immunology for improving the health of children with allergic and immune conditions through innovative research, outstanding clinical care, and education of the current and next generation of leaders in healthcare and research

\section{DISCLOSURE}

RCO has research funding from Takeda Pharmaceuticals and Astra Zeneca. SPH is a consultant with Immune Pharmaceuticals. MER has an equity interest in reslizumab (Teva Pharmaceuticals), is a consultant for Immune Pharmaceuticals, is the inventor of several eosinophilic esophagitis-related patents owned by Cincinnati Children's Hospital Medical Center, is on the American Partnership for Eosinophilic Disorders (APFED) Medical Advisory Board, and is on the Steering Committees of the International Eosinophil Society (IES) and The International Gastrointestinal Eosinophil Researchers (TIGERS). The remaining authors declare no conflict of interest.

C 2014 Society for Mucosal Immunology

\section{REFERENCES}

1. Mulder, D.J. \& Justinich, C.J. Understanding eosinophilic esophagitis: the cellular and molecular mechanisms of an emerging disease. Mucosal Immunol. 4, 139-147 (2011).

2. Liacouras, C.A. et al. Eosinophilic esophagitis: updated consensus recommendations for children and adults. J. Allergy Clin. Immunol. 128, 3-20. e26; quiz 21-22 (2011).

3. Abonia, J.P. \& Rothenberg, M.E. Eosinophilic esophagitis: rapidly advancing insights. Annu. Rev. Med. 63, 421-434 (2012).

4. Parfitt, J.R., Gregor, J.C., Suskin, N.G., Jawa, H.A. \& Driman, D.K. Eosinophilic esophagitis in adults: distinguishing features from gastroesophageal reflux disease: a study of 41 patients. Mod. Pathol. 19, 90-96 (2006).

5. Ravelli, A.M. et al. Dilated intercellular spaces: a major morphological feature of esophagitis. J. Pediatr. Gastroenterol. Nutr. 42, 510-515 (2006).

6. Mueller, S, Neureiter, D, Aigner, T \& Stolte, M. Comparison of histological parameters for the diagnosis of eosinophilic oesophagitis vs. gastrooesophageal reflux disease on oesophageal biopsy material. Histopathology 53, 676-684 (2008).

7. Ingerski, L.M. et al. Health-related quality of life across pediatric chronic conditions. J. Pediatr. 156, 639-644 (2010).

8. Menard-Katcher, P, Marks, K.L., Liacouras, C.A., Spergel, J.M., Yang, Y.X \& Falk, G.W. The natural history of eosinophilic oesophagitis in the transition from childhood to adulthood. Aliment. Pharmacol. Ther. 37, 114-121 (2013).

9. Harris, R.F., Menard-Katcher, C, Atkins, D, Furuta, G.T. \& Klinnert, M.D. Psychosocial dysfunction in children and adolescents with eosinophilic esophagitis. J. Pediatr. Gastroenterol. Nutr. 57, 500-505 (2013).

10. Blanchard, C. et al. Eotaxin-3 and a uniquely conserved gene-expression profile in eosinophilic esophagitis. J. Clin. Invest. 116, 536-547 (2006).

11. Blanchard, C. et al. IL-13 involvement in eosinophilic esophagitis: transcriptome analysis and reversibility with glucocorticoids. J. Allergy Clin. Immunol. 120, 1292-1300 (2007).

12. Simpson, C.L., Patel, D.M. \& Green, K.J. Deconstructing the skin: cytoarchitectural determinants of epidermal morphogenesis. Nat. Rev. Mol. Cell Biol. 12, 565-580 (2011).

13. Hanakawa, Y. et al. Differential effects of desmoglein 1 and desmoglein 3 on desmosome formation. J. Invest. Dermatol. 119, 1231-1236 (2002).

14. Getsios, S. et al. Desmoglein 1-dependent suppression of EGFR signaling promotes epidermal differentiation and morphogenesis. J. Cell Biol. 185, 1243-1258 (2009).

15. Chen, Y.J. et al. DSG3 facilitates cancer cell growth and invasion through the DSG3-Plakoglobin-TCF/LEF-Myc/Cyclin D1/MMP signaling pathway. PLOS ONE 8, e64088 (2013).

16. Brown, L. et al. Desmoglein 3 promotes cancer cell migration and invasion by regulating activator protein 1 and protein kinase C-dependent-Ezrin activation. Oncogene advance online publication, 10 June 2013; doi:10.1038/onc.2013.186 (e-pub ahead of print).

17. Mannan, T, Jing, S, Foroushania, S.H., Fortune, F \& Wan, H. RNAimediated inhibition of the desmosomal cadherin (desmoglein 3) impairs epithelial cell proliferation. Cell Prolif. 44, 301-310 (2011).

18. Green, K.J. \& Simpson, C.L. Desmosomes: new perspectives on a classic. J. Invest. Dermatol. 127, 2499-2515 (2007).

19. Green, K.J. \& Gaudry, C.A. Are desmosomes more than tethers for intermediate filaments?. Nat. Rev. Mol. Cell Biol. 1, 208-216 (2000).

20. Harmon, R.M. et al. Desmoglein-1/Erbin interaction suppresses ERK activation to support epidermal differentiation. J. Clin. Invest. 123, 1556-1570 (2013)

21. Shirakata, $Y$, Amagai, M, Hanakawa, $Y$, Nishikawa, T \& Hashimoto, $K$. Lack of mucosal involvement in pemphigus foliaceus may be due to low expression of desmoglein 1. J. Invest. Dermatol. 110, 76-78 (1998).

22. Tunggal, J.A. et al. E-cadherin is essential for in vivo epidermal barrier function by regulating tight junctions. EMBO J. 24, 1146-1156 (2005).

23. Kalabis, J. et al. Isolation and characterization of mouse and human esophageal epithelial cells in 3D organotypic culture. Nat. Protoc. 7, 235-246 (2012).

24. Frankart, A, Malaisse, J, De Vuyst, E, Minner, F, de Rouvroit, C.L. \& Poumay, Y. Epidermal morphogenesis during progressive in vitro 3D reconstruction at the air-liquid interface. Exp. Dermatol. 21, 871-875 (2012).

25. Andl, C.D. et al. Epidermal growth factor receptor mediates increased cell proliferation, migration, and aggregation in esophageal keratinocytes in vitro and in vivo. J. Biol. Chem. 278, 1824-1830 (2003).

26. Oyama, K. et al. AKT induces senescence in primary esophageal epithelial cells but is permissive for differentiation as revealed in organotypic culture. Oncogene 26, 2353-2364 (2007).

27. Michaylira, C.Z. et al. Periostin, a cell adhesion molecule, facilitates invasion in the tumor microenvironment and annotates a novel tumor-invasive signature in esophageal cancer. Cancer Res. 70, 5281-5292 (2010).

28. Shirakawa, Y. et al. Topological analysis of p21WAF1/CIP1 expression in esophageal squamous dysplasia. Clin. Cancer Res. 6, 541-550 (2000).

29. Ohkawa, T. etal. Localization of heparanase in esophageal cancer cells: respective roles in prognosis and differentiation. Lab. Invest. 84, 1289-1304 (2004). 
30. Amagai, M, Matsuyoshi, N, Wang, Z.H., Andl, C \& Stanley, J.R. Toxin in bullous impetigo and staphylococcal scalded-skin syndrome targets desmoglein 1. Nat. Med. 6, 1275-1277 (2000).

31. Rodrigo, S. et al. High intraepithelial eosinophil counts in esophageal squamous epithelium are not specific for eosinophilic esophagitis in adults. Am. J. Gastroenterol. 103, 435-442 (2008).

32. Orlando, L.A. \& Orlando, R.C. Dilated intercellular spaces as a marker of GERD. Curr. Gastroenterol. Rep. 11, 190-194 (2009).

33. Blanchard, C. et al. Coordinate interaction between IL-13 and epithelial differentiation cluster genes in eosinophilic esophagitis. J. Immunol. 184, 4033-4041 (2010)

34. Zuo, L. et al. IL-13 induces esophageal remodeling and gene expression by an eosinophil-independent, IL-13R alpha 2-inhibited pathway. J. Immunol. 185, 660-669 (2010).

35. Wex, T. et al. Gastro-oesophageal reflux disease is associated with up-regulation of desmosomal components in oesophageal mucosa. Histopathology 60, 405-415 (2012).

36. Brennan, D. et al. Differential structural properties and expression patterns suggest functional significance for multiple mouse desmoglein 1 isoforms. Differentiation 72, 434-449 (2004).

37. Hatano, Y. et al. The Th2 cytokine, interleukin-4, abrogates the cohesion of normal stratum corneum in mice: implications for pathogenesis of atopic dermatitis. Exp. Dermatol. 22, 30-35 (2013).

38. Blanchard, C. et al. A striking local esophageal cytokine expression profile in eosinophilic esophagitis. J. Allergy Clin. Immunol. 127, 208-217, e201-207 (2011).

39. Vicario, M. et al. Local B cells and IgE production in the oesophageal mucosa in eosinophilic oesophagitis. Gut 59, 12-20 (2010).

40. Tsukita, S, Furuse, $M \&$ Itoh, M. Multifunctional strands in tight junctions. Nat. Rev. Mol. Cell Biol. 2, 285-293 (2001).

41. Yoshida, K, Yokouchi, M, Nagao, K, Ishii, K, Amagai, M \& Kubo, A. Functional tight junction barrier localizes in the second layer of the stratum granulosum of human epidermis. J. Dermatol. Sci. 71, 89-99 (2013).

42. Furuse, M. et al. Claudin-based tight junctions are crucial for the mammalian epidermal barrier: a lesson from claudin-1-deficient mice. J. Cell Biol. 156, 1099-1111 (2002).

43. Kirschner, N, Rosenthal, R, Furuse, M, Moll, I, Fromm, M \& Brandner, J.M. Contribution of tight junction proteins to ion, macromolecule, and water barrier in keratinocytes. J. Invest. Dermatol. 133, 1161-1169 (2013).

44. Monkemuller, K. et al. Role of tight junction proteins in gastroesophageal reflux disease. BMC Gastroenterol. 12, 128 (2012).

45. Kubo, A, Nagao, K, Yokouchi, M, Sasaki, H \& Amagai, M. External antigen uptake by Langerhans cells with reorganization of epidermal tight junction barriers. J. Exp. Med. 206, 2937-2946 (2009).

46. Lucendo, A.J. et al. Immunophenotypic characterization and quantification of the epithelial inflammatory infiltrate in eosinophilic esophagitis through stereology: an analysis of the cellular mechanisms of the disease and the immunologic capacity of the esophagus. Am. J. Surg. Pathol. 31, 598-606 (2007).

47. Yen, E.H. et al. Comparative analysis of FcepsilonRl expression patterns in patients with eosinophilic and reflux esophagitis. J. Pediatr. Gastroenterol. Nutr. 51, 584-592 (2010).

48. Bannert, C. et al. Fc-epsilon-RI, the high affinity lgE-receptor, is robustly expressed in the upper gastrointestinal tract and modulated by mucosal inflammation. PLOS ONE 7, e42066 (2012).

49. Mulder, D.J., Pooni, A, Mak, N, Hurlbut, D.J., Basta, S \& Justinich, C.J. Antigen presentation and MHC class II expression by human esophageal epithelial cells: role in eosinophilic esophagitis. Am. J. Pathol. 178, 744-753 (2011).

50. Blanchard, C. et al. Periostin facilitates eosinophil tissue infiltration in allergic lung and esophageal responses. Mucosal Immunol. 1, 289-296 (2008).

51. Jia, G. et al. Periostin is a systemic biomarker of eosinophilic airway inflammation in asthmatic patients. J. Allergy Clin. Immunol. 130, 647-654 e610 (2012).

52. Masuoka, M. et al. Periostin promotes chronic allergic inflammation in response to Th2 cytokines. J. Clin. Invest. 122, 2590-2600 (2012).

53. Johansson, M.W., Annis, D.S. \& Mosher, D.F. alpha(M)beta(2) integrinmediated adhesion and motility of IL-5-stimulated eosinophils on periostin. Am. J. Resp. Cell Mol. Biol. 48, 503-510 (2013).

54. Rothenberg, M.E. et al. Common variants at $5 \mathrm{q} 22$ associate with pediatric eosinophilic esophagitis. Nat. Genet. 42, 289-291 (2010).
55. Sherrill, J.D. et al. Variants of thymic stromal lymphopoietin and its receptor associate with eosinophilic esophagitis. J. Allergy. Clin. Immunol. 126, 160-165 e163 (2010).

56. Takayama, G. et al. Periostin: a novel component of subepithelial fibrosis of bronchial asthma downstream of IL-4 and IL-13 signals. J. Allergy Clin. Immunol. 118, 98-104 (2006).

57. Yan, W \& Shao, R. Transduction of a mesenchyme-specific gene periostin into 293T cells induces cell invasive activity through epithelialmesenchymal transformation. J. Biol. Chem. 281, 19700-19708 (2006).

58. Kalluri, R \& Neilson, E.G. Epithelial-mesenchymal transition and its implications for fibrosis. J. Clin. Invest. 112, 1776-1784 (2003).

59. Kagalwalla, A.F. et al. Eosinophilic esophagitis: epithelial mesenchymal transition contributes to esophageal remodeling and reverses with treatment. J. Allergy Clin. Immunol. 129, 1387-1396 e1387 (2012).

60. Muir, A.B. et al. Esophageal epithelial and mesenchymal cross-talk leads to features of epithelial to mesenchymal transition in vitro. Exp. Cell Res. 319, 850-859 (2013).

61. Lin, M.S. et al. Desmoglein-1-specific T lymphocytes from patients with endemic pemphigus foliaceus (fogo selvagem). J. Clin. Invest. 105, 207-213 (2000).

62. Simon, D, Hoesli, S, Roth, N, Staedler, S, Yousefi, S \& Simon, H.U. Eosinophil extracellular DNA traps in skin diseases. J. Allergy Clin. Immunol. 127, 194-199 (2011).

63. Smith, D.L., Smith, J.G., Wong, S.W. \& deShazo, R.D. Netherton's syndrome: a syndrome of elevated IgE and characteristic skin and hair findings. J. Allergy Clin. Immunol. 95 (1 Pt 1), 116-123 (1995).

64. Chavanas, S. et al. Mutations in SPINK5, encoding a serine protease inhibitor, cause Netherton syndrome. Nat. Genet. 25, 141-142 (2000).

65. Descargues, P. et al. Spink5-deficient mice mimic Netherton syndrome through degradation of desmoglein 1 by epidermal protease hyperactivity. Nat. Genet. 37, 56-65 (2005).

66. Deraison, C. et al. LEKTI fragments specifically inhibit KLK5, KLK 7, and KLK14 and control desquamation through a pH-dependent interaction. Mol. Biol. Cell 18, 3607-3619 (2007).

67. Bonnart, C. et al. Elastase 2 is expressed in human and mouse epidermis and impairs skin barrier function in Netherton syndrome through filaggrin and lipid misprocessing. J. Clin. Invest. 120, 871-882 (2010).

68. Samuelov, L. et al. Desmoglein 1 deficiency results in severe dermatitis, multiple allergies and metabolic wasting. Nat.Genet. 45, 1244-1248 (2013).

69. Lu, T.X. et al. MicroRNA signature in patients with eosinophilic esophagitis, reversibility with glucocorticoids, and assessment as disease biomarkers. J. Allergy Clin. Immunol. 129, 1064-1075 e1069 (2012).

70. Langmead, B, Trapnell, C, Pop, M \& Salzberg, S. L. Ultrafast and memoryefficient alignment of short DNA sequences to the human genome. Genome Biol. 10, R25 (2009).

71. Trapnell, C, Hendrickson, D.G., Sauvageau, M, Goff, L, Rinn, J.L. \& Pachter, L. Differential analysis of gene regulation at transcript resolution with RNA-seq. Nat. Biotechnol. 31, 46-53 (2013).

72. Garber, M, Grabherr, M.G., Guttman, M \& Trapnell, C. Computational methods for transcriptome annotation and quantification using RNA-seq. Nat. Methods 8, 469-477 (2011).

73. Harada, H. et al. Telomerase induces immortalization of human esophageal keratinocytes without p16INK4a inactivation. Mol. Cancer Res. 1, 729-738 (2003).

74. Vath, G.M. et al. The structure of the superantigen exfoliative toxin A suggests a novel regulation as a serine protease. Biochemistry 36, 15591566 (1997).

75. Hanakawa, Y. et al. Molecular mechanisms of blister formation in bullous impetigo and staphylococcal scalded skin syndrome. J. Clin. Invest. 110, 53-60 (2002).

76. Ishii, K, Harada, R, Matsuo, I, Shirakata, Y, Hashimoto, K \& Amagai, M. In vitro keratinocyte dissociation assay for evaluation of the pathogenicity of anti-desmoglein $3 \mathrm{lgG}$ autoantibodies in pemphigus vulgaris. J. Invest. Dermatol. 124, 939-946 (2005).

77. Jovov, B, Que, J, Tobey, N.A., Djukic, Z, Hogan, B.L. \& Orlando, R.C. Role of $\mathrm{E}$-cadherin in the pathogenesis of gastroesophageal reflux disease. Am. J. Gastroenterol. 106, 1039-1047 (2011).

78. Wu, D. et al. Interleukin-13 (IL-13)/IL-13 receptor alpha1 (IL-13Ralpha1) signaling regulates intestinal epithelial cystic fibrosis transmembrane conductance regulator channel-dependent $\mathrm{Cl}$ - secretion. J. Biol. Chem. 286, 13357-13369 (2011) 